\title{
Terminal QoS of Adaptive Applications and its Analytical Computation
}

\section{Pratyush Moghé}

Network and Service Management Research Dept., Bell Labs. pmoghe@bell-labs.com

Asawaree Kalavade

DSP \& VLSI Systems Research Dept., Bell Labs.

kalavade@bell-labs.com

\begin{abstract}
Next-generation slim hosts or terminals (like PDA's, settop boxes, and network computers) are expected to support sophisticated continuous media applications. Since these terminals have very limited processing power, the application processing delay can be a significant component of the end-to-end QoS. We call this delay a terminal QoS measure to distinguish it from end-to-end QoS measures like packet loss, delay, and delay jitter. Computing the processing delay can be tricky since many of the continuous media applications are smart and can adapt to network conditions. Such applications can use RTP/RTCP feedback of end-to-end QoS to continually change their output bitrate. Each adaptation is associated with a different amount of processing on the terminal.

In this context, we pose the following problem. Consider a terminal that supports multiple concurrent adaptive applications. Each application uses end-toend QoS feedback to adapt itself according to its adaptation algorithm. What effect do the adaptations have on the processing demand on the terminal? That is, what is the processing delay as a function of the adaptations? In this paper, we provide a theoretical framework to understand this interaction.
\end{abstract}

\section{Keywords}

QoS Modeling-Analysis-Evaluation, Adaptive Applications, Internet Application Management, QoS Guarantees in Internet, Terminal or Host QoS, Continuous-Media Applications.

\section{INTRODUCTION}

There is an intensive research and development effort ongoing in trying to make slim hosts like PDA's, settop boxes, and communicators Internet-aware 
[1]. Such hosts have limited processing capability but are being used to support increasingly sophisticated applications. In the scope of this paper, we call such hosts terminals, to distinguish them from the hosts that connote a PC or workstation with larger processing capability and memory. The next generation terminals are expected to support continuous-media (CM) applications such as audio and video streams, along with other traditional applications. Currently such CM applications are supported on powerful hosts [2]. Many of these applications are implemented and delivered using the RTP transport framework [5]. A few [2,3] have also been improvised to adapt their behavior based on the feedback of RTCP messages that convey end-to-end QoS parameters like packet loss, network delay, etc. It is expected that such adaptive CM applications will eventually be deployed on terminals. Under such a scenario, we conjecture that the processing delay on end terminals could become a significant part of the end-to-end QoS. Processing delay on the terminals is thus a QoS measure. We prefer to call it a measure of terminal QoS to distinguish it from end-to-end QoS.

If we analyze recent work on QoS-based issues, it is evident that there are two distinct threads, one that focuses on the networking aspects and the other on the operating systems (OS) aspects. The philosophy of the first thread is to ensure end-to-end QoS in the face of network evils. The basic idea is to give applications a controlled share of the network resources so as to safeguard their performance. Typically, QoS is ensured through a combination of reservation protocols [4] and transport delivery/adaptation/renegotiation mechanisms [5]. For instance, network protocols like RSVP can be used to specify and guarantee resources within the network while frameworks like RTP/RTCP can be used to regulate the rate of a sending application so as to reduce network traffic. The other community focuses on the OS aspects of end-systems, and aims to provide processing guarantees to $\mathrm{CM}$ applications. This is typically done through the use of real-time priority-based schedulers and clever resource sharing schemes $[6,7,8]$.

Given the relatively mature body of work in both these communities, it is appropriate to start exploring the interaction between their work. For instance, in the networking community, the design of RTP-based adaptation algorithms has thus far been based on end-to-end QoS measures such as loss $[3,5,9]$. In such algorithms, when the packet loss increases beyond a threshold, the adaptation algorithm shifts to lower adaptation levels. A lower adaptation level corresponds to a reduced bit-rate and hence lower network traffic. In general, changing adaptation levels changes the processing demand on the terminal, thus changing* the terminal QoS (quantified by the overall processing delay of an application). Thus far, there has been little work in characteriz-

*Table 1 in [10] presents a good example of how the CPU execution time increases with lowered adaptation level in the context of packet audio. LPC encoding (lower adaptation level) in an audio application incurs 110 times the execution time as PCM encoding (higher adaptation level). 
ing the impact of adaptations on the total processing delay of an application, especially when multiple such applications concurrently share the processor.

From the other end, studies in the multimedia and real-time OS community have designed run-time schedulers to support applications with varying load. However, specific changes in applications due to adaptations have not been studied. Also, we are not aware of any work that analyzes (at compile or design time) the impact of adaptive applications on processing.

In this paper, we wish to address this intersecting hole. Our objective is to quantify the impact of multiple adaptive applications on the terminal QoS (processing delay) of each application. Refer to Figure 1. This is a hard problem since adaptive applications introduce variability in the offered processing. Our approach will be abstract simple models (based on a mixture of related work in the literature and a good deal of intuition!) for adaptive CM applications and for the processing demands on a terminal. Assuming these models, we derive exactly the total processing delay distribution of each application when multiple adaptive applications concurrently run on the terminal. We expect the results obtained in the paper to be used to design adaptation algorithms, real-time schedulers, and to understand clearly the tradeoff between end-to-end QoS and terminal QoS.

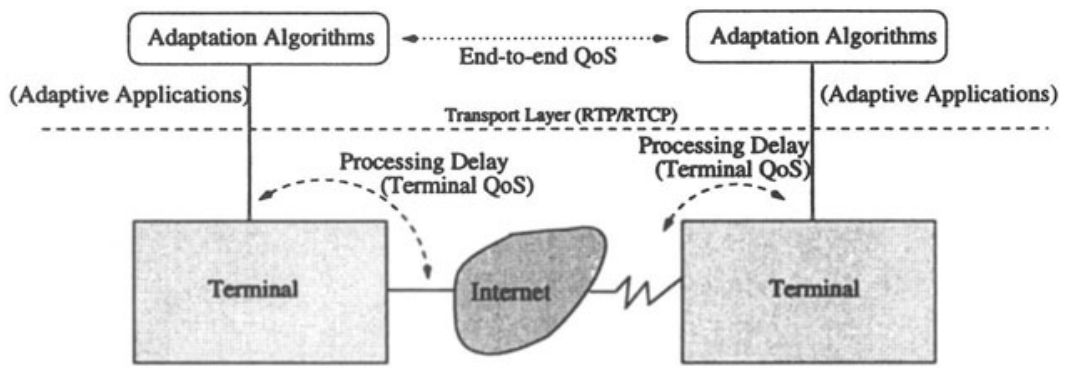

Figure 1 End-to-end QoS and Terminal QoS

\subsection{Methodology}

We now summarize the proposed methodology - details are presented through the rest of the paper. The first step is to model the adaptation of each application at the sender and receiver terminals as a time-varying dynamic process. The notion is that each adaptive application is associated with different adaptation levels, where an adaptation level represents a specific bit-rate. This bit-rate could correspond to a particular refresh rate [3, 9], or a particular algorithm [10], or to a particular coded layer [11]. We focus on modeling the adaptation of applications based on RTP/RTCP loss feedback. In this case, the adaptation process of an application changes its level when the next feed- 
back report (RTCP sender/receiver report) indicates that the network loss exceeds a certain threshold. We model the transitions of this adaptation process through time-invariant probabilities. The interval between reports is assumed to be uniformly distributed (in accordance with the interval of RTCP reports [5], assuming none of them are lost!). In the second step, we model each application as a task graph with several alternative execution paths. Each task can have an arbitrarily distributed execution time. A run-time environment activates the tasks to be run on the processor based on a priority function; tasks are nonpreemptive. We assume that only one of the several execution paths in the application task graph is activated at run-time depending on the current adaptation level. This provides the link between the adaptation in the application and its computation demand. Unfortunately, this dependence makes the analysis of the computation complicated. We invoke a reasonable assumption that the task-level changes (rate of milliseconds) are much faster than the changes in the adaptation process (rate of seconds or minutes); consequently, we can characterize the state of computation on the processor between two consecutive changes in the adaptation process. In the third step, we consider multiple applications that run concurrently on the terminal. Each application is assumed to adapt based on its adaptation process. We show that the processing demand imposed by a particular vector of adaptation levels can be analyzed by formulating the computation as a well-defined stochastic process. Our main result is that we derive exactly the processing delay distribution in terms of the stationary distribution of the jumps made by the computation process.

The rest of the paper is organized as follows. In Section 2, we describe how applications are specified. In Section 3, we propose models for the adaptation process and for the computation process. In Section 4 we analyze the computation process by formulating it as a semi-Markov process, and we compute its stationary distribution. In Section 4.2, we derive the processing delay distribution. We conclude after outlining possible applications of our analytical results.

\section{APPLICATION SPECIFICATION}

We assume that each application is specified by a task graph consisting of nodes and arcs as shown in Figure 2a. Nodes represent task-level computations and arcs specify precedences between nodes. Each node in the graph (other than the source and sink nodes) is assumed to incur an arbitrarily distributed execution time on its designated resource. Without loss of generality, we assume that the execution time is a discrete random variable [12] that takes one of the values $e_{1}, e_{2}, . ., e_{k}$ with probabilities $p_{1}, p_{2}, . ., p_{k}$ respectively, where these probabilities sum to 1 . Such a distribution can approximately model execution time variations due to data dependencies or due to architectural effects like cache misses and I/O contention. 


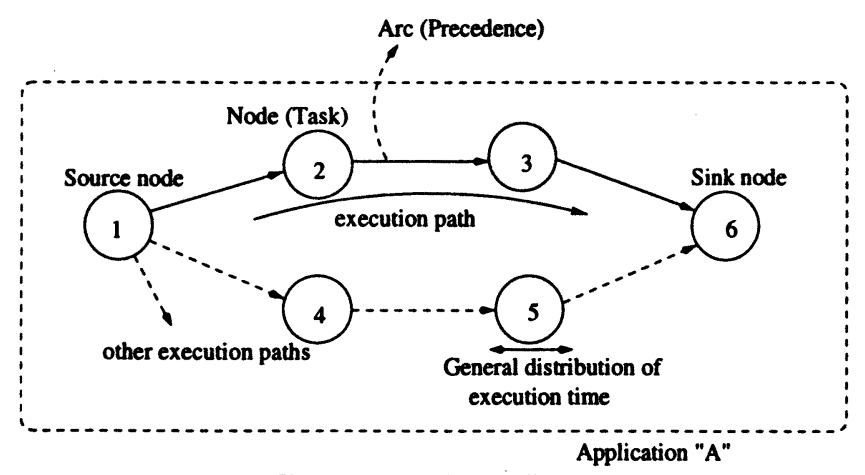

a. Single iteration of the application

Application "A"

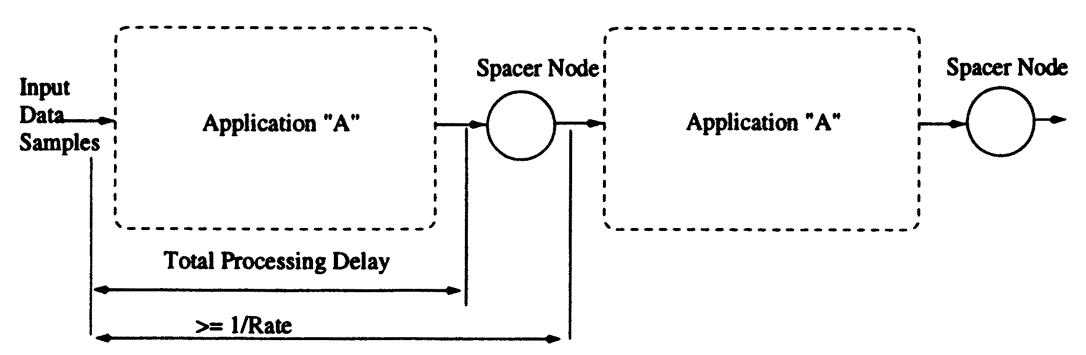

b. Repetitive behavior of the application is specified by successive iterations, separated by a spacer node

Figure 2 Application Specification and Repetitive Behavior

An application consists of several alternative execution paths within each iteration. Each path is made up of a succession of nodes between the source and sink nodes. An execution path is generally assumed to be chain structured. When multiple arcs merge into a node, we assume that the node waits for execution till it receives data from all its incident arcs that are included in the current execution path.

To model the repetitive behavior of $\mathrm{CM}$ applications, we assume that data samples arrive at the (infinitely buffered) source node of an application at a fixed interarrival frequency dictated by the input rate of the application. For instance, in a video application this rate could correspond to the refresh rate; if the refresh rate is $\mathbf{3 0}$ frames/sec, the interarrival time is $33 \mathrm{~ms}$. The precise time when these input samples are taken in by the source node for processing depends on when the earlier samples have finished processing. If the earlier samples are still being processed by the application when the next input samples arrive, the input samples are made to wait, otherwise, they are taken in for processing immediately. This behavior is modeled in the application task graph by the use of an artificial node called the spacer node. Refer to Figure $2 \mathrm{~b}$. Thus, samples are made to wait if the processing delay of the earlier samples is less than the interarrival time between samples; the spacer 
node releases samples at exactly the interarrival frequency. If, on the other hand, the processing delay is more than interarrival interval, the spacer node releases samples immediately after the processing is done. In either case, the application task graph repeats as soon as the spacer node releases the samples. This models the behavior of repetitive continuous media applications.

As stated before, the current level of the adaptation process of the application determines the execution path to be activated within the current iteration of the application. These concepts are now clarified by means of an example. Figure 3 shows the select parts of an H.261 video encoder application [13]. We indicate only a single iteration of the application for lack of space. Two alternative execution paths, each with different time intervals available for processing are shown. The two paths correspond to refresh rates of say $15 \mathrm{fps}$ and $8 \mathrm{fps}$. One of the two paths is activated at the beginning of each iteration depending on the latest adaptation level. The fork in each execution path after the quantizer indicates that data samples traverse both the arcs to the VLE and the IQuantizer.

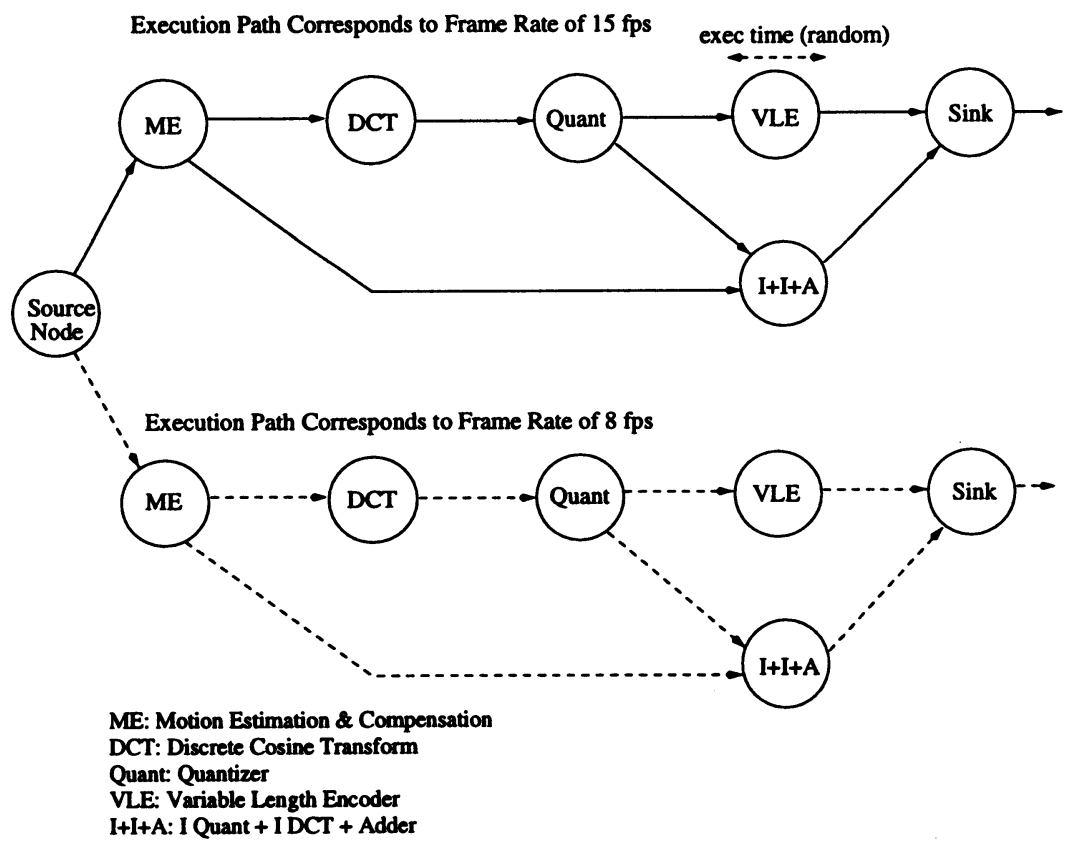

Figure 3 Specifying an iteration of the H.261 video encoder application

Note that an audio encoder could be described similarly. Unlike video though, the input rate of audio samples is typically constant. In this case, an adaptive audio encoder can have different execution paths represent different encoding algorithms, not different refresh rates. The adaptation levels 
and the most recent RTCP feedback can be used to switch between different encoding algorithms like PCM, ADPCM, GSM, or their combinations, etc.

Thus far, we have modeled a single application. To model multiple concurrent applications, we combine the task graphs of individual applications into a single aggregated graph. Since applications can begin at different starting times, their temporal skew is captured by the addition of a node to each application that models the lag of each application. The aggregated graph terminates into a final sink node when all the individual applications finish their processing at the same exact time. The processing delay is measured over the interval of the aggregated graph.

\section{ANALYTICAL MODELS}

In this section, we discuss the analytical models used to describe the adaptation process of each application and the state of computation in a terminal that runs multiple adaptive applications.

\subsection{Adaptation Process}

We consider the particular case when applications adapt based on RTCP-like feedback of network congestion parameters such as packet loss or delay. This feedback occurs through sender (or receiver) reports. We assume that the inter-report time $T_{\text {report }}^{A}$ to an adaptive application is uniformly distributed between minimum and maximum limits of $t_{\text {report, min }}^{A}$ and $t_{\text {report,max }}^{A}$ respectively [5]. Once a report is received, the current adaptation level is either retained or changed depending on the end-to-end QoS feedback. We model the adaptation level changes at the receipt of each report as Markovian jump process with level-dependent transitions. Thus we approximate the possibility of different QoS reports in a level by different probabilities. Refer to Figure 4. The transitions between adaptation levels $m$ and $n$ are assumed to occur with a probability $v_{m n}$. This probability can be determined by profiling the adaptation of an actual application under an adaptation algorithm. ([3] represents an example, where the adaptation is based on an algorithm that imposes an additive increase, a multiplicative decrease, with a small dead-zone to deduce the maximum output rate of a sender.) Note that these transition probabilities are time-homogeneous; this may not be a valid approximation in practice, but as a starting point, we believe it is reasonable.

Assuming each application adapts based on its reported end-to-end QoS, independent of other applications, we can construct similar models for all the concurrent applications being supported on a terminal. If $M_{t}^{i}$ represents the adaptation level of application $i$ at instant $t$, the joint process $M=$ $\left\{M_{t}^{1}, M_{t}^{1}, . ., M_{t}^{i}, ..\right\}$ represents the joint adaptation level of all the applications currently running on the terminal. 


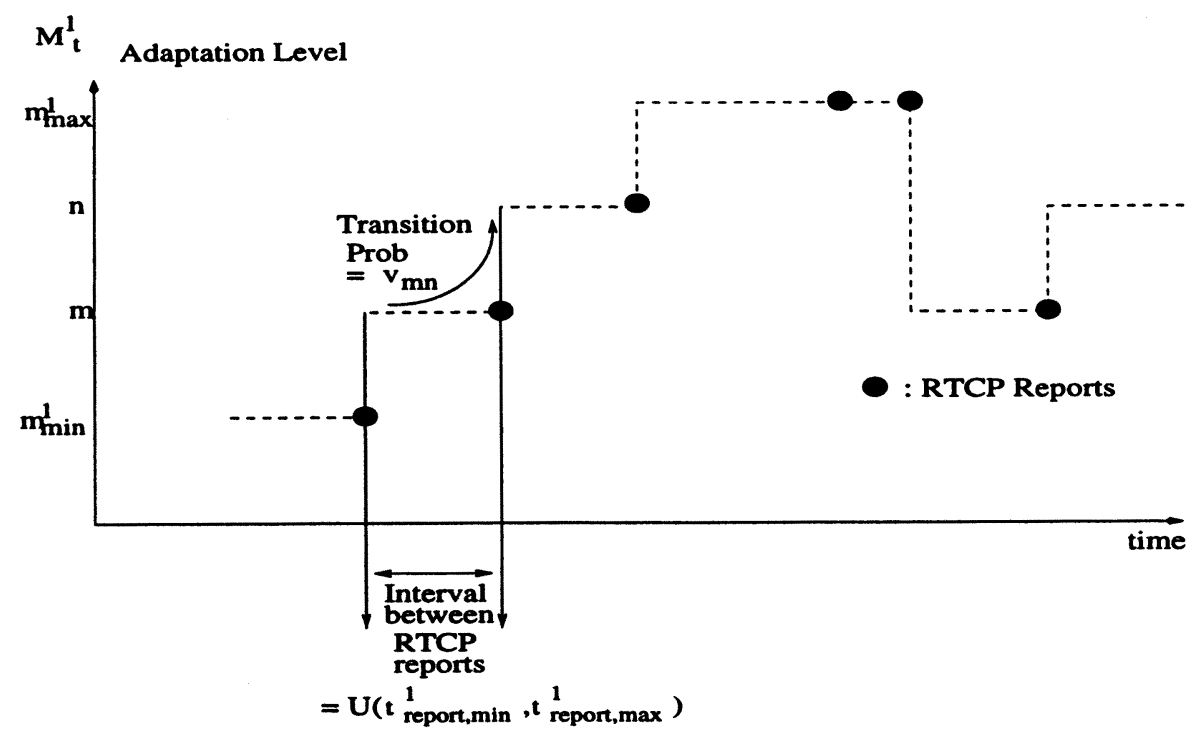

Figure 4 Modeling the adaptation process of application 1

\subsection{Computation Process}

In this section, we are interested in characterizing the processing load that is offered to the terminal by multiple adaptive applications. We make the following assumptions regarding the run-time environment in the terminal: (1) Mapping of tasks to the processor is done at compile time and is known. (2) Tasks are non-preemptive. (3) A simple run-time scheduler allocates one of the possibly many waiting tasks to a ready processor based on a known priority function. (4) Communication costs are ignored, as are effects of limited memory.

As discussed before, changes in the adaptation level of each application trigger changes in the execution path of the application. Naturally, the processing load changes when the adaptation level changes. It is extremely hard to analyze changes in the processing load coupled with the changes in the adaptation level. Fortunately, we can use the property that changes in the adaptation level are on a much larger time-scale than the changes in the processing due to different tasks. Under the circumstances, we can assume that the aggregate processing load on the terminal reaches steady-state between the consecutive changes in the joint adaptation process. As we shall see, this simplifies the analysis considerably, and allows us to decouple the dynamics of the processing load from the dynamics of the adaptation process.

We now represent the state of computation in the terminal through a state process. The key observation is that we can model the state process as a semiMarkov stochastic process. Such a process has only one-step memory and the stationary probability distribution of its jumps to a particular state can be 
exactly computed. This in turn enables us to compute the processing delay of an application. In Section 4.2, we derive the processing delay distribution.

We define next a few variables that characterize the state of computation as a function of time. Let the transition sequence $A=\left\{A_{n}, n=0,1, ..\right\}$ be the time-instants when the state of the computation changes. $A_{0} \triangleq 0$. We define a vector sequence $Y=\left\{Y_{n}, n=0,1, ..\right\}$, where $Y_{n}=\left(I, w_{I}\right)=$ (set of nodes ready and waiting at transition time $A_{n}^{-}$, the delay incurred by these nodes thus far), where $A_{n}^{-}$denotes the time just prior to the $n$th transition time. Next, we define the sequence $Z=\left\{Z_{n}, n=0,1, ..\right\}$, where $Z_{n}=\left(J, r_{J}\right)$ $=$ (set of nodes running at transition time $A_{n}$, remaining execution time of these nodes). Sequences $Y$ and $Z$ capture information about running and waiting nodes. We also define a sequence $U$ that stores, at discrete points in time, information about how long an application path has been running. That is, $U=\left\{U_{n}, n=0,1, ..\right\}$, where $U_{n}=\left(A p p, m^{A p p}, t^{A p p}\right)=$ (application, adaptation level of application at instant $A_{n}^{-}$, elapsed time of application at time $\left.A_{n}^{-}\right)$. Here, $t^{A p p}$ is set to zero at the start of each application iteration, and is incremented at transitions when nodes in that iteration finish running or finish waiting. Figure 5 illustrates the state process for a simple example with two applications.

Observation The joint sequence $(Y, Z, U)$ represents the state of computation at each transition and is a Markov chain. By joining the state of computation between transitions by straight lines, we obtain the state process $X . X$ is a continuous-time stochastic process and since its underlying jump process $(Y, Z, U)$ is Markovian, it is a semi-Markov process* [14]. We omit the proof for brevity.

\section{COMPUTING TERMINAL QOS}

In this section, we derive the terminal QoS measures based on the framework developed thus far. In the first subsection, we compute, for a given vector $\mathcal{M}$ of adaptation levels, the stationary distribution $\pi_{i j a}^{\mathcal{M}}$ of the sequence $(Y, Z, U)$, where $(i, j, a)$ represents a state visited by $(Y, Z, U)$. Here, $i, j$, and $a$ are shorthand for $\left(I, w_{I}\right),\left(J, r_{J}\right)$, and $\left(A p p, m^{A p p}, t^{A p p}\right)$ respectively. Recognize that this distribution is conditioned on a particular value of $\mathcal{M}$. Assuming that the adaptation process converges to a steady-state distribution, we show that we can obtain an unconditioned stationary distribution $\pi$. In the second subsection, we use this distribution to derive the processing delay distribution of an application.

* $X$ is NOT a Markov chain since it does not spend an exponentially distributed time in each of its states. 


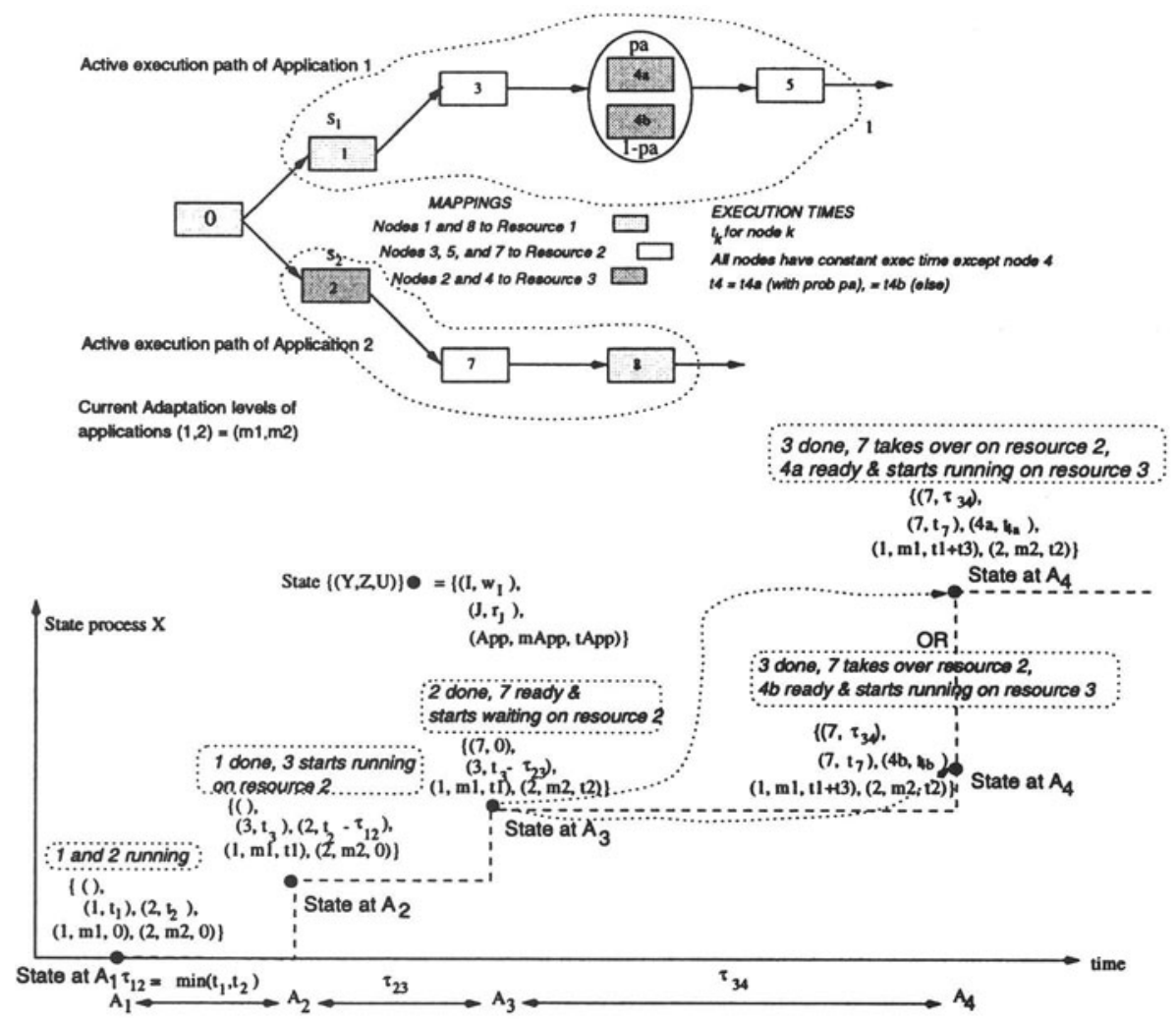

Figure 5 State Process

\subsection{Stationary distribution of $(Y, Z, U)$}

We have stated earlier that, for a particular adaptation value $\mathcal{M}$, the joint process $(Y, Z, U)$ is a discrete-time Markov chain. This means that when $(Y, Z, U)$ jumps from state $(i, j, a)$ to state $(k, l, c)$, its future evolution is independent of the past, given state $(i, j, a)$. This chain is therefore completely specified in terms of its transition probability function defined by $R_{i j a}^{k l c} \triangleq$ $P\left\{\left(Y_{n+1}, Z_{n+1}, U_{n+1}\right)=(k, l, c) \mid\left(Y_{n}, Z_{n}, U_{n}\right)=(i, j, a)\right\},(i, j, a),(k, l, c) \in S$, where $S$ is the state space of $X$. $R_{i j a}^{k l c}$ is the probability that $(Y, Z, U)$ moves from a state $(i, j, a)$ to the state $(k, l, c)$ in a single jump. This one-step probability can be determined for a particular set of applications.

It can be shown that $R$ has a unique stationary distribution $\pi^{\mathcal{M}}$, under fairly general conditions [14]. The stationary distribution $\pi_{i j a}^{\mathcal{M}}$ is the probability that $(Y, Z, U)$ jumps to state $(i, j, a)$, given that $(Y, Z, U)$ changes states. The stationary distribution $\pi^{\mathcal{M}}$ can be computed from $R$, since it satisfies the linear equations: $\sum_{(i, j, a) \in S} \pi_{i j a}^{\mathcal{M}} R_{i j a}^{k l c}=\pi_{k l c}^{\mathcal{M}}, \forall(k, l, c) \in S$, and $\sum_{(k, l, c) \in S} \pi_{k l c}^{\mathcal{M}}=1$.

To obtain the stationary distribution unconditioned of adaptation level $\mathcal{M}$, 
we assume that the adaptation process converges to a steady-state distribution $\gamma$. Here, $\gamma_{\mathcal{M}}$ is roughly the long-term fraction of time the adaptation process spends in level $\mathcal{M}$. $\gamma$ can be computed easily from the transition matrix of the adaptation process and the interval of the RTCP reports. We omit the details for lack of space. Assuming $\gamma$ is determined, the unconditioned stationary distribution $\pi$ is $\pi_{i j a}=\sum_{\forall \mathcal{M}} \pi_{i j a}^{\mathcal{M}} \times \gamma_{\mathcal{M}}$.

\subsection{Processing Delay Distribution}

Suppose we wish to determine the probability that the processing delay of a particular execution path $m$ of an application exceeds delay $\delta$. Let node $b$ be the predecessor to the spacer node on the execution path $m$. Then, $\operatorname{Pr}\{$ Processing delay of path $m>\delta\} \triangleq \max _{\mathcal{M}}[\operatorname{Pr}\{$ Processing delay of path $m>\delta, m \in \mathcal{M}\}$ ]. Note that the right side of this equation is a $\max$ operator over all adaptation vectors of which path $m$ is an element.

The expression inside the max operator is the ratio of the number of times the processing delay of the path $m$ exceeds $\delta$ to the number of times path $m$ is activated. Recall that $\pi_{i j a}^{\mathcal{M}}$ represents the probability of a jump of the underlying process $(Y, Z, U)$ to the state $(i, j, a)$, conditioned on a jump. If we focus only on the jumps to state where node $b$ begins running, it is clear that:

$\operatorname{Pr}\{$ Processing delay of path $m>\delta, m \in \mathcal{M}\}=\frac{\sum_{\forall i j a} \pi_{i j a}^{\mathcal{M}} \mid b \in J, r_{b}=t_{b}, t_{A p p}+r_{b}>\delta}{\sum_{\forall i j a} \pi_{i j a}^{\mathcal{M}} \mid b \in J, r_{b}=t_{b}}$ Having computed the processing delay of path $m$ in the application, we can compute the processing delay of the entire application by simply choosing the worst-case processing delay among all its execution paths.

Other terminal QoS measures like average nodal wait and average processing delay can be easily computed from our analysis.

\section{CONCLUSIONS}

We are still in the process of obtaining numerical results based on this theoretical framework. We expect to use the derived processing delay to determine the maximum (or minimum) sustainable adaptation level for an acceptable processing delay. Terminal QoS can also be used to tune the adaptation algorithms. Currently each application has its native adaptation algorithm that operates independently of other applications, their adaptation algorithms, or the scheduler. Finally, we hope to use the results here to quantify the interaction between end-to-end QoS and terminal QoS.

To conclude, we note that this is only a preliminary step toward exploring the interaction between adaptations (driven by end-to-end QoS) and terminal QoS. We intend to examine the validity of our models by means of actual measurements. 


\section{REFERENCES}

[1] PDA Buyer's Guide, Pen Computing Magazine, vol. 3, no. 11, July/August 1996, pp. 84.

[2] T. Turletti, "The INRIA Videoconferencing System (IVS)", ConneXions, vol. VIII, no. 10, Oct. 1994.

[3] I. Busse, B. Deffner, H. Schulzrinne, "Dynamic QoS Control of Multimedia Applications based on RTP", Computer Communications, Vol. 19, No. 1, Jan. 1996, pp. 49-58.

[4] R. Braden, ed., and L. Zhang, et al., "Resource Reservation Protocol (RSVP) - Version 1 Functional Specification," draft-ietf-rsvp-spec-14.ps, Nov. 1996.

[5] H. Schulzrinne, S. Casner, R. Frederick, V. Jacobson, "RTP: A Transport Protocol for Real-Time Applications", RFC 1889, Audio-Video Transport Working Group, January 1996.

[6] C. L. Liu and J. W. Layland, "Scheduling Algorithms for Multiprogramming in a Hard Real Time Environment", JACM, 20(1):46-61, 1973.

[7] K. Ramamritham, et al., "Scheduling Algorithms and Operating Systems Support for Real-Time Systems", Proceedings of the IEEE, vol. 82, no. 1, Jan. 1994, pp. 55-66.

[8] D. K.Y. Yau and S. S. Lam, "Adaptive Rate-Controlled Scheduling for Multimedia Applications", Proc. ACM Multimedia'96, Boston, MA, Nov. 1996.

[9] J.-C. Bolot, T. Turletti, "A Rate Control Mechanism for Packet Video in the Internet", Proc. IEEE Infocom '94, Toronto, Canada, June 1994, pp. 1216-23.

[10] J.-C. Bolot, A. Vega-Garcia, "Control Mechanisms for Packet Audio in the Internet", Proc. IEEE Infocom '96, San Francisco, CA, April 1996, pp. 2329.

[11] S. McCanne, M. Vetterli, V. Jacobson, "Receiver-driven Layered Multicast", Proc. of SIGCOMM'96, Stanford, CA, Aug. 1996, pp. 117-130.

[12] Jong Kim, K. G. Shin, "Execution Time Analysis of Communicating Tasks in Distributed Systems", IEEE Transactions on Computers, vol. 45, no. 5, May 1996, pp. 572-9.

[13] V. Bhaskaran, K. Konstantinides, Image and Video Compression Standards: Algorithms and Architectures, Kluwer Academic Publishers, 1995.

[14] E. Cinlar, Introduction to Stochastic Processes, Prentice-Hall, Englewood Cliffs, 1975.

\section{BIOGRAPHY}

Pratyush Moghé (pronounced: Prat-yoosh Mo-gay) is a Member of Technical Staff in the Network and Service Management Research Department at Bell Labs. He received the Ph.D. from UCLA in 1995. His activities focus on the design and prototyping of Internet application management toolkits. He devotes his spare time to music and to his $300 \mathrm{ZX}$.

Asawaree Kalavade is a Member of Technical Staff in the DSP and VLSI Systems Research Department at Bell Labs, where she currently works on tools for the codesign of high-performance multimedia systems. Asawaree received the Ph.D. in EECS from UC Berkeley in 1995. 